DOE/MC/29115-96/C0634

Image Processing Algorithm Design and Implementation for Real Time Autonamous Inspection of Mixed Wastes

Authors:

$$
\text { CONF-960368--1 }
$$

R.J. Schalkoff

K.M. Shaaban

A.E. Carver

\title{
Contractor:
}

South Carolina Universities Research Foundation (SCURF)

Strom Thurmond Institute

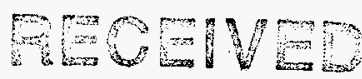

Clemson, South Carolina 29634

Contract Number:

DE-AC21-95MC29115

Conference Title:

1996 Symposium on Visual Communications and Image Processing

\section{Conference Location:}

Orlando, Florida

\section{Conference Dates:}

March 17-20, 1996

\section{Conference Sponsor:}

The International Society for Optical Engineering 


\section{DISCLAIMER}

Portions of this document may be illegible in electronic image products. Images are produced from the best available original document. 


\section{DISCLAIMER}

This report was prepared as an account of work sponsored by an agency of the United States Government. Neither the United States Government nor any agency thereof, nor any of their employees, makes any warranty, express or implied, or assumes any legal liability or responsibility for the accuracy, completeness, or usefulness of any information, apparatus, product, or process disclosed, or represents that its use would not infringe privately owned rights. Reference herein to any specific commercial product, process, or service by trade name, trademark, manufacturer, or otherwise does not necessarily constitute or imply its endorsement, recommendation, or favoring by the United States Government or any agency thereof. The views and opinions of authors expressed herein do not necessarily state or reflect those of the United States Government or any agency thereof.

This report has been reproduced directly from the best available copy.

Available to DOE and DOE contractors from the Office of Scientific and Technical Information, 175 Oak Ridge Turnpike, Oak Ridge, TN 37831; prices available at (615) 576-8401.

Available to the public from the National Technical Information Service, U.S. Department of Commerce, 5285 Port Royal Road, Springfield, VA 22161; phone orders accepted at (703) 487-4650. 


\title{
Image Processing Algorithm Design and Implementation for Real-Time Autonomous Inspection of Mixed Waste*
}

\author{
Robert J. Schalkoff, Khaled M. Shaaban and Albrecht E. Carver \\ Dept. Electrical and Computer Engineering \\ Clemson University \\ Clemson, SC 29634-0915 \\ (803)-656-5913 (voice) \\ (803)-656-7220 (FAX) \\ rjschal@clemson.edu
}

KEYWORDS:

Color image segmentation, HSI processing, texture detection, image processing hardware

\begin{abstract}
The ARIES \#1 vision system is used to acquire drum surface images under controlled conditions and subsequently perform autonomous visual inspection leading to a classification as 'acceptable' or 'suspect'. Specific topics described include vision system design methodology, algorithmic structure, hardware processing structure, and image acquisition hardware. Most of these capabilities were demonstrated at the ARIES Phase II Demo which was held on November 30,1995 . Finally, Phase III efforts are briefly addressed.
\end{abstract}

\section{Introduction}

ARIES (Autonomous Robotic Inspection Experimental System), is under development for the Department of Energy to survey and inspect drums containing low-level radioactive waste stored in warehouses at DOE facilities. Large quantities of low-level radioactive waste are stored in steel drums at various Department of Energy (DOE) sites in the United States. Much of the stored waste qualifies as mixed waste and falls under Environmental Protection Agency (EPA) regulations that require periodic inspection. The general rules governing the storage of hazardous waste are spelled out in the Code of Federal Regulations ${ }^{1}$. The rules that have specific implications regarding the inspection process may be summarized as follows:

1. The owner operator must inspect often enough to identify and correct problems before harm is done (265.15).

2. Records must be kept of the inspection process and must include a specified minimum amount of information

This work was funded by the U. S. Department of Energy Morgantown Energy Technology Center, Contract METC DE-AC2192MC29115.

${ }^{1}$ U. S. Environmental Protection Agency, Regulations of Hazardous Waste, 40 CFR 265, Federal Register, 1992. 
(265.15).

3. Stored containers must be examined at least weekly to look for leaks and deterioration caused by corrosion or other factors (265.174).

The robot will navigate through the aisles and perform an inspection operation, typically performed by a human operator, making decisions about the condition of the drums and maintaining a database of pertinent information about each drum. The drums are typically stacked four high and arranged in rows with three-foot aisle widths. The system will navigate through the aisles and, on the basis of visual information, make autonomous decisions about the condition of the drums and maintaining a database of pertinent information about each drum. This system will locate and identify each drum, locate any unique visual features, characterize relevant surface features of interest (such as paint blisters, rusted areas, etc.), and update a database containing the inspection data. An adaptive algorithm and learning concept, requiring little effort by unskilled operators, is used to "train" the vision system prior to the actual inspection process.

A camera positioning system includes primitive instructions for the robot to reference and position the vision payload, and retracts to a more compact position when traveling in the open warehouse. During inspection, the mechanical deployment system extends up and folds out to deploy the four inspection packages at different heights on the four- drum stacks. The stacked drums may be 55-, 85-, and 110-gallon capacity in a given column.

The overall system is shown in Figure 1.



Figure 1: ARIES System 


\section{Vision System Requirements}

\subsection{Objectives}

Visual assessment of drum condition is an autonomous assessment of visible and quantifiable surface characteristics based upon available image data. The problem is essentially a two-class risk minimization problem, ${ }^{\text {Sch92 }}$ where drums are classified as "acceptable" or "suspect." A drum would be considered "suspect" if it exhibits sufficient surface deterioration to warrant warning of possible failure. The system should err on the conservative side i.e., the system should rarely miss a "suspect" drum, whereas misclassification of a good drum as "suspect,", while inconvenient, is not as significant.

Based on expert opinions, the surface blemishes which indicate probable drum failure are rust patches on the order of 0.5 " $x 0.5$ " and paint blisters (indicating internal rust). The human inspector usually relies on the characteristic color of rust in classifying it, hence this was the obvious feature to utilize in segmenting rust patches. Likewise, the human uses patterns of the reflections from a blistered surface in classifying it. This suggested that the vision system could rely upon texture segmentation for classifying these regions. Detecting these features requires a color camera with sufficient resolution to resolve the texture elements of blisters and lighting with consistent color temperature and direction (with respect to the camera).

\subsection{Processing Time and Power}

The required inspection rate of one drum every two seconds requires considerable computational power ${ }^{2}$. This is shown in Figure 2. This constraint severly limits the amount of processing available per drum. Other constraints

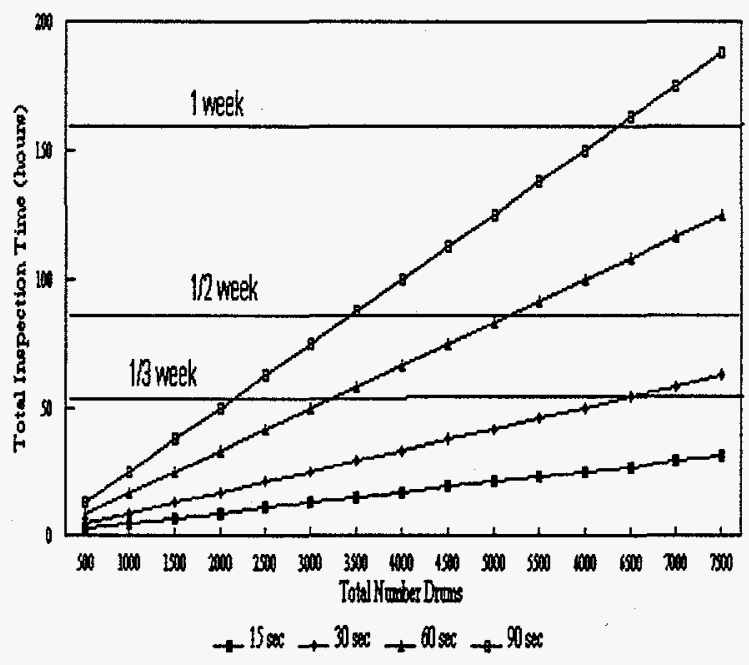

Figure 2: Inspection Capacity as Function of Single Drum Inspection Time

include minimal size, weight, and power consumption.

\footnotetext{
${ }^{2}$ which usually, given the temporal processing constraints, translates into electrical power.
} 
Current temporal processing capability allows all vision operations in $\approx 3 \mathrm{sec}$. per half-drum ( 1 image), i.e., $6 \mathrm{sec}$ per drum. Further speedup is possible, given faster strobe recharge times and further algorithm recoding.

\section{Principal Algorithms}

Processing of acquired image data occurs in two main steps. The images(s) are segmented to ascertain that a drum, or drum portion, exists in the sensor field of view (FOV). If a drum is found, the size and extent of the drum is computed (using active vision) and the images are subsequently classified by region. Therefore, classifying a drum on the basis of passive visual information is carried out in four steps:

1. segmenting the drum (section) from the total imaged scene, i.e., finding the image region corresponding to only the visible part of the drum in the image(s)

2. segmenting rust regions,

3. segmenting paint blisters, and

4. overall classification and recording of results.

\subsection{Segmentation Algorithms}

Segmentation is the process of finding a connected region within the image with a specific property such as color or intensity, or a relationship between pixels, that is, a pattern. Classification of a drum as "suspect" is done if the number of pixels denoting rusty regions or paint blisters exceeds a specific threshold. The threshold is tunable depending site-specific requirements. Since drum failure modes and human inspector assessments appear to be highly site-specific, it was deemed necessary that the algorithms should be adaptable to the site requirements. A learning algorithm is provided which, given inputs from a human "tutor", adjusts the algorithm parameters. It is straightforward to add additional feature extraction approaches to the present system, once suitable decompositions into hardware-implementable computations are designed.

\subsection{Drum Segmentation}

Due to warehouse imaging conditions, segmentation of the imaged drum from the scene background (which consists of, among other entities, other similar drums) is a challenging task. Traditional edge extraction and region segmentation techniques fail due to a variety of nonideal working conditions (glossy drums, multiple reflections, gradual fading of intensity, etc.).

Another aspect of the drum segmentation is the exclusion of regions on the drum which are not to be analyzed for rust or blisters. These regions are paper labels such as barcodes and warning signs typically found on the drums. The diffuse reflections from paper is typically much greater than that from the paint. Since diffuse reflections are direction insensitive, the paper is segmented from the drum by finding regions which exhibit less change in intensity when the lighting direction is changed.

From the specification of the problem and the capabilities of the navigation/camera positioning system, the following assumptions are employed in segmenting the drum from the scene:

- The warehouse will contain only three drum sizes. 
- Each stack of drums will contain a single drum size.

- Each drum has a homogeneous paint color. However, there is no restrictions on or requirement for apriori knowledge of the specific color.

- Projected laser dots are easily discernible on flat-painted surface.

- Specular reflections are easily discernible on glossy-painted surfaces.

- The height of the camera with respect to the floor is known.

- The distance to the drum surface from a camera will be within the range of 24 " to 54 ".

- An image will contain the horizontal center and either the top or bottom edge of a drum.

- The dominant color of the visible region of a drum is its paint color (barcodes and other labels do not dominate the scene).

The technique that was developed utilizes the above assumptions and can be decomposed into a sequence of 'bootstrapped' steps: finding the drum center and distance; finding a rough estimate of the vertical edges; finding the top or bottom edge; picking a drum size; and then refining the estimate of the vertical edges. Each of these steps is described below.

The process of finding the center of the drum starts by imaging the laser dots projected onto the surface of the drum. Using apriori knowledge of the laser/camera geometry, estimates of the three dimensional location of the dots are found. For each combination of 3 dots, the virtual vertical cylinder on which the dots lie is found. Any of these cylinders whose radius or location are not within the expected values corresponding to one of the three drum sizes and the known imaging geometry are excluded from further analysis. The set of projections of the virtual cylinder centers on the image plane will be refered to as the center-set.

In the case of glossy painted drums, the projected laser dots are much harder to detect since the majority of the laser energy is reflected specularly away from the camera. This reduces the reliability of the laser-center-finding technique. However, if the drum generates strong specular reflections, then the location of the specular spots from judiciously located lamps can be used to locate the center of the drum in the image. If the lights are vertically in line with the camera, specular reflections can only occur on surfaces for which the horizontal component of the surface normal vector is pointing at the camera. Since the drums are vertically oriented cylinders, only the vertical stripe closest to the camera, hence at the center of the drum image, will be oriented so that specular reflections can be seen. By isolating specular reflections matching the expected reflections from the lamps, candidate centers are determined and included in the center-set.

The drum center is then determined as the mean of the largest cluster of estimated centers (a subset of centerset) whose span is less than a predetermined bound. Using this drum center and location of the laser dot closest to this center, the distance to the surface of the drum is estimated via geometry. Likewise, a rough estimate of the locations of the drum's vertical edges is obtained, assuming a 55-gallon drum.

By restricting the analysis region to be within the rough estimates of the drum's vertical edges, it is safe to assume that the drum dominates the analysis region. Thus the ranges of hue, saturation, and intensity which characterize the drum can be determined via histograms. By selecting the pixels within these ranges, a binary image is constructed which represents the drum in the scene. This binary image is then compared to a set of previously generated set of templates. Each element in this set is a binary image which represents a drum whose top or bottom is at a known location in the image. Thus, the template which best matches the binary image provides the location of the actual top or bottom.

Knowing the vertical height of a drum top or bottom, the height of the camera, and the heights of the different drum sizes and pallets, the size of the drum is easily deduced. Once the drum size is known, the estimate of the locations of the drum's vertical edges can be improved, which in turn defines the final analysis region. 


\subsection{Rust Segmentation}

\subsubsection{Color Space Fundamentals}

One of the obvious properties of a rust region is its color, therefore segmentation using pixel color as the characteristic element is used. Typically, the output of color video cameras are in a format which makes detecting colors, independent of variations in other parameters such as intensity, difficult. To facilitate the segmentation, the images are converted to a hue-saturation-intensity (HSI) color representation. In this color space, a color image is represented by the three gray-level images, referred to as planes.

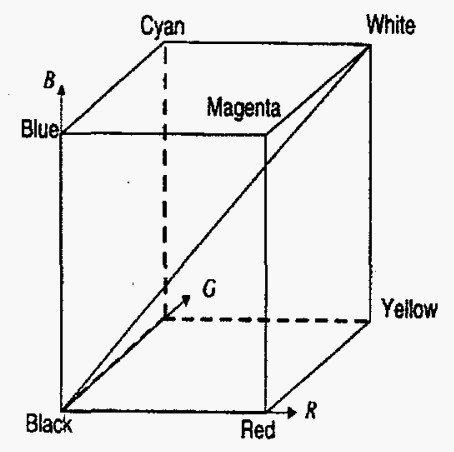

Figure 3: RGB Coordinate System

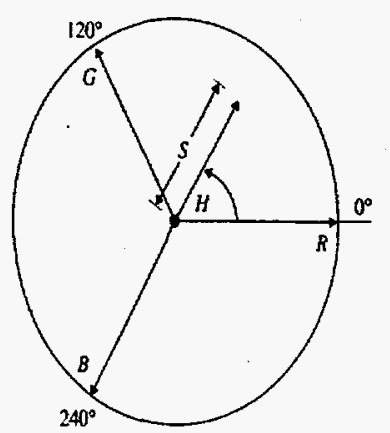

Figure 4: Hue Space Color Map Example (Red reference at $=$ $0 \mathrm{deg})$

In the hue plane, a pixel value is a numeric representation of the color. In the saturation plane, a pixel value is the purity of the color (high values indicate pure colors whereas low values indicate substantial mixing with white light). In the intensity plane, a pixel value denotes the brightness. ${ }^{\mathrm{Jai} 89}$ In the implementation of HSI space, each pixel is represented by three values between 0 and 255. For the saturation and intensity, 0 represents the minimum values and 255 represents the maximum values. For the hue, the zero-reference is cyan and greens are around 45 , reds are around 130 , and blues are around 210 .

\subsubsection{Application to Drum Images}

In training data, rust was found to exhibit hue values typically between 90 and 160 . It was also found that rust has a saturation value less than 60 . Since this range is dependent on camera white balance, gamma corrections, and the lighting color temperature, this range must be established for any differences in these parameters. A pixel is considered a rust pixel if its hue and saturation falls within both of these ranges. Note, however, that if a pixel has either a high intensity or low saturation value, the hue information is unreliable. Considering this, the basic element for rust segmentation is a pixel with a hue value within the range of 90 to 160 , a saturation value within the range of 12 to 60 , and an intensity value less than 200 .

Labeling all of the rust pixels is performed by thresholding the three planes over the ranges specified above and then "ANDING" the results. Connecting the rust pixels is the process of finding regions in which the density of rust pixels exceeds a predetermined threshold value. The density of rust associated with a given pixel is defined to be the number of rust pixels in a neighborhood of that pixel. A pixel with 9 or more rust pixels in its $4 \times 4$ neighborhood is considered a member of a rusty region. 


\subsection{Paint Blister Segmentation}

A paint blister is a conglomeration of several small, almost circular bubbles, protruding from the surface. Under a controlled lighting conditions (intensity and geometry), the image of the light reflected off of one of these bubbles is a relatively consistent spatial intensity pattern. This suggested that the paint blister segmentation could rely on a spatial basic element. From frequency analysis over a set of training images it was found that if a pixel, $x(i, j)$, in the intensity plane satisfies:

$$
\left(\begin{array}{cc}
x(i, j)-x(i-\Delta i, j)>30 & \text { AND } \\
x(i, j)-x(i+\Delta i, j)>30 & \text { AND } \\
x(i, j)-x(i, j-\triangle j)>30 & \text { AND } \\
x(i, j)-x(i, j+\Delta j)>30 & \text { AND } \\
x(i, j)>50 &
\end{array}\right)
$$

this pixel should be classified as part of a paint bubble. Depending upon the camera and optics specification (focal length, CCD ship size, resolution, etc.), $\triangle_{i}$ and $\triangle_{j}$ are chosen to optimize performance. Labeling the bubble pixels is performed by co-occurrence analysis with this pattern and connecting is performed as in the rust segmentation process.

\section{Learning}

Each of the vision algorithms used requires a set of operating parameters which directly affects system performance. To optimize this performance, an adaptation or optimization procedure to generate the optimum parameter set is required. Unfortunately, the problem cannot be directly formulated as a general nonlinear optimization problem because of the lack of a suitable quantitative performance measure or objective function. Instead of attempting to generate or estimate this performance measure, an interactive, operator-guided approach is used.

The operator interface used for system training has the form of a multi-window display. Each of the windows contains the resulting image from the segmentation algorithm using a specific set of parameters. The operators task is to only compare between windows to determine the relative quality of the results. The Nelder-Mead algorithm (Downhill Simplex Method) is the optimization procedure used. It requires a comparison of the objective function values at a limited number of search points $(\mathrm{N}+1$, in $\mathrm{N}$-dimension search space). For this algorithm, the operator need only decide the best and the worst images presented in the multi-window display. The algorithm uses the operator's decision to determine a new search "point" and hence a new set of images, derived from updated parameters, to present to the operator. This procedure continues until a satisfactory result is obtained.

\section{Algorithm Implementation}

\subsection{Processing System}

Since system speed is an all-important constraint, specialized image processing/computer vision hardware is used to implement the aforementioned segmentation algorithms.

The vision industry is following two design strategies, one in which hardware is tailored for specific vision processing tasks, and the other in which general DSP chipset is utilized to provide more generic capabilities. The 
former strategy provides for greater performance if one's algorithm can be decomposed into a series of subtasks implementable by the hardware. The latter strategy compromises speed for computational flexibility. The Aries vision system falls within the first category.

Aries \#1 employs a modular vision system, manufactured by Imaging Technology Inc. (ITI), with full scale pipeline processing capabilities. The system uses two ITI IMA150/40 memory managers, each with four Mbytes of reconfigurable memory. These cards are designed to carry submodules that perform different image processing operations. One of its design features is a multi-input-multi-output cross-port switch that allows the reconfiguration of the pipeline for different operations. The current system uses two ITI IMA150/40 memory managers, each with four Mbytes of reconfigurable memory.

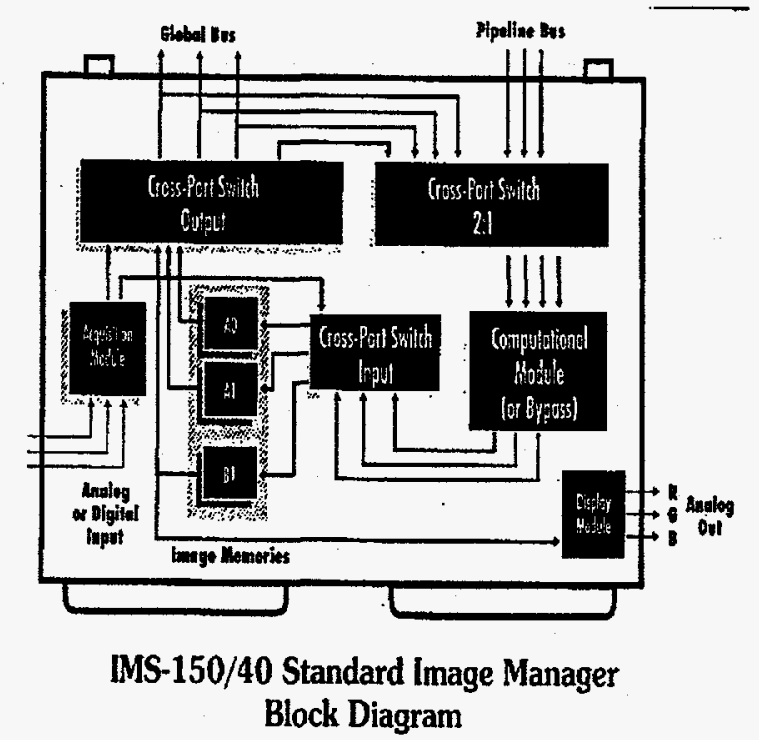

Figure 5: ITI IMA

\subsubsection{Image Processing Hardware}

The following submodules are used:

1. Acquisition Module : The acquisition module is used for digitizing a true color images (24 bits) from RGB PAL camera at 25 frame/sec. The resolution used is $(768 \times 572)$ which leads to a digitization rate of $(768 \times 572 \times 3 \times 25=32.94 \mathrm{Mbytes} / \mathrm{sec})$. Also this module performs the conversion of the cameras RGB output to the needed HSI color space in real time.

2. Convolver/Arithmetic Logic Unit This is the main module used for most of the vision tasks required for image segmentation. This includes convolutions, thresholding, rank filtering and connecting. This module also carries a statistical processor which is used to count the number of pixels that belong to each class during the segmentation process.

3. Histogram/ Feature Extraction Processor This processor is used to perform the necessary histogram operation required for identifying the drum color. The module is also used to generate both vertical and horizontal projections of the image which is necessary to locate the drum in the acquired image. 


\subsection{Image Acquisition System and Hardware}

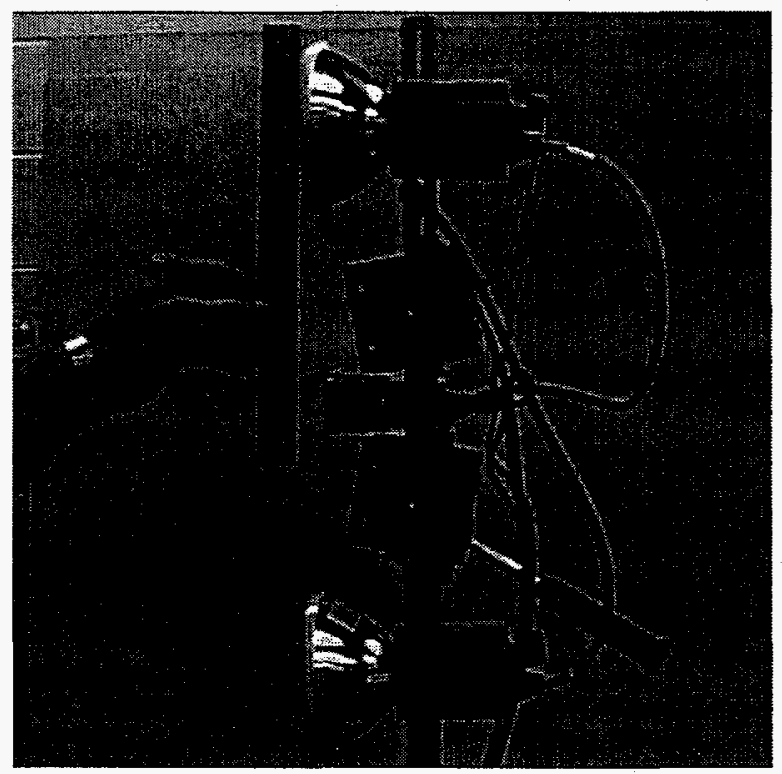

Figure 6: Basic Imaging Head (Dot Projector Not Shown)

\subsubsection{Passive Components}

The imaging system includes four camera subsystems, one for each drum in a stack of four. It also includes two digitizing modules which provide the ability to digitize images from all four cameras. Each camera subsystem consists of a camera with one strobe lamp above it and one below it. It also incorporates a five-dot laser structuredlight source. The expected variability in site ambient lighting and power limitations of the mobile robot led to the development of a strobe-based image acquisition system. The camera used is a Panasonic GP-US502E, three CCD high resolution color camera.

To gain more vertical resolution, the version of the camera was chosen to follow the CCIR PAL standard. In this standards the camera provides 572 lines versus the 480 lines specified in the RS-170 NTSC standards commonly used in the USA. A single PAL image, referred to as a frame, consists of two interlaced fields separated in time by 0.02 seconds. To prevent "striping" in the image, one must provide consistent lighting to both of these fields. Since we are using strobe lighting, a single flash must be provided for each field and synchronized to the that field (specifically at the start of the CCD integration time).

Utilizing the memory management card internal flags which indicate the start of the next field, and a custom strobe control circuit, we are able to synchronize any strobe lamp to a field. However, since a single strobe has a recharge time on the order of two seconds, we are unable to provide the lighting for two successive fields (one frame) with a single strobe lamp. Even though the fields are lighted at the appropriate time, since the two strobes are spatially separated, the resultant lighting is not consistent in both fields yielding "striped" images. The software-solution to this problem is addressed next. 


\subsubsection{Illumination}

Each camera system consists of a camera with one strobe lamp above it and one below it. Since a single image acquired by the camera will have inconsistently illuminated fields due to the spatial separation of the lamps, two images are taken, one in which the first field is lit by the upper lamp while the second is lit by the lower and another in which the first field is lit by the lower lamp and the second by the upper. Combining the first field from the first image and the second field from the second image results in an image in which both fields are lit by the upper lamp. Similarly, an image lit by the lower lamp is generated. A technique using the ITI Convolver/Arithmetic-Unit module has been developed to accomplish this process at real-time rates.

\section{Additional Algorithms Developed}

During Phase II, several ancillary vision algorithms were developed. Several of these will be considered for implementation in Phase III. Specific algorithms were:

- Real Time Drum Center Detection Using Active Vision. This capability will be fused with the sonar-based drum location algorithms and allows more precise positioning of the vision system.

The objective of this algorithm is to find the center of the drum while the robot is moving. This operation is necessary for the robot to stop in the right position with the stack of drums to be analyzed in the center of the camera's field of view. Currently this operation is done using a sonar system. Adding this capability of the vision system may improve the performance of the sonar or it may be used to replace it if proven to be more reliable.

The system utilizes one of the cameras and laser dot projectors. This projector is located about 10 inches above the center of the camera pointing down such that the dot is within the field of view for the anticipated working ranges. This camera-laser arrangement allows a rough estimate for the range between the camera point-of-projection and the reflection of the laser dot on the surface of the drum. The smaller the range is, the higher the location of the projection of laser dot on the image plane. Thus, as the robot moves, the laser dot moves across the surface of the drum changing the range from maximum at the edge of the drum to a minimum at the center to again a maximum at the other end. This change in range can be detected from the projection of the laser dot in the image plane. Based upon this idea, the following algorithm was developed in order to find the center of the drum:

1. Acquire an image with laser on.

2. Locate the laser dot in the image if it exists.

3. Depending upon the location of the dot in this image compared to the previous image we may have one of the following three events:

- UP: The location of the projection of the dot went up which implies a reduction in the range.

- DOWN: The location of the projection of the dot went down which implies an expansion in the range.

- FLAT: The location of the projection of the dot did not change which implies no change in the range.

4. If the laser dot is crossing the drum while acquiring a sequence of frames we should expect a sequence of events having the form:

$$
[U P, U P, \cdots, F L A T, F L A T \cdots, D O W N, D O W N, \cdots]
$$

The occurrence of this pattern is a good indication that the laser dot has passed across a drum. 


\section{Summary}

Planned efforts for Phase III include:

- Commercialization of the Phase II prototype vision system.

- Incorporation of additional features,and alternative algorithms, as appropriate and feasible.

- Acquisition of additional real warehouse drum data for training and testing purposes.

- Possible implementation of vision software using an alternative computing platform and operating system.

- Fielding of the system at a DOE site, and comparision of system performance with that of human inspectors.

\section{REFERENCES}

[BS95] A. Busboom and R.J. Schalkoff. Direct surface parameter estimation using structured light: A predictor-corrector based approach. Image and Vision Computing, Accepted Sept. 1995.

[Jai89] A.K. Jain. Fundamentals of Digital Image Processing. Prentice-Hall, 1989.

[Sch89] R.J. Schalkoff. Digital Image Processing and Computer Vision. John Wiley and Sons, 1989.

[Sch92] R. J. Schalkoff. Pattern Recognition: Statistical, Structural and Neural Approaches. John Wiley, 1992.

[SKH+93] R.J. Schalkoff, M. Kraess, C. Herwig, K. Shaaban, and R.H. Finley. A modular and extendible image processing system: Update to version IP6.1 (including DOE inspection effort). Technical Report TR-080693-0915-I, Dept. of Electrical and Computer Engineering, Clemson University, August 1993. 
The implementation of this idea assumed that the speed of the robot is defined by range of maximum and minimum expected speeds. Accordingly, the algorithm was designed to tolerate variations of the robot speed. A more accurate version of this algorithm could be developed if we have an accurate information about the instantaneous speed (or location) of the robot.

- Barcode Detection via Textural Analysis. This capability was carried over from Phase I, and implemented using the ITI hardware. In Phase III, the possibility of reading barcodes via the vision system sensors, as opposed to freestanding barcode readers, will be investigated.

- An Efficient and Accurate Algorithm for Direct Measurement of Cylindrical Surface Parameters. This is described in detail in. ${ }^{\text {BS95 }}$ The basic idea is to project a horizontal line pattern onto the object, which is assumed to be a cylinder. A passive image is acquired by a camera and some characteristic coordinates are extracted from the image of the stripes. These points are then used for an initial estimation of the position, orientation and size of the imaged object, resulting in an initial set of surface parameters. The position of the characteristic points for a projection of the same light pattern on the object given by the estimated surface parameters is computed. The position, location and size features of this "virtual" cylinder are estimated, using the same methods like for the actual image. The differences between the features computed from actual and estimated / virtual object are used for an affine transform of the estimated cylinder parameters. This transform results in an updated, improved set of surface parameters describing the object. The resulting virtual passive image is again computed and the correction step repeated as necessary. Finally the real image taken by the camera and the virtual image should match and the surface parameters of the object be very accurately estimated by the surface parameters obtained by the successive affine transform steps.

The Table below shows sample results using this algorithm.

Table 1: Results from ICA Experiments for Cylinders

\begin{tabular}{|c|c|c|c|c|c|c|c|}
\hline \multicolumn{8}{|c|}{ ICA Feature Results for Cylinder 1: } \\
\hline Feature & True Value & $\begin{array}{c}\text { Sim. } \\
\text { Result }\end{array}$ & $\begin{array}{c}\text { abs.Sim. } \\
\text { Error }\end{array}$ & $\begin{array}{c}\text { rel.Sim. } \\
\text { Error }\end{array}$ & $\begin{array}{l}\text { Exp. } \\
\text { Result }\end{array}$ & $\begin{array}{c}\text { abs.Exp. } \\
\text { Error }\end{array}$ & $\begin{array}{c}\text { rel.Exp. } \\
\text { Error }\end{array}$ \\
\hline $\begin{array}{c}y \\
z \\
\text { radius } \\
\theta_{\mathrm{y}} \\
\theta_{\mathrm{z}} \\
\end{array}$ & $\begin{array}{r}0.0000 \\
47.2500 \\
11.2500 \\
0.000^{\circ} \\
0.000^{\circ}\end{array}$ & $\begin{array}{r}0.0000 \\
47.1716 \\
11.2193 \\
0.000^{\circ} \\
0.000^{\circ} \\
\end{array}$ & $\begin{array}{r}0.0000 \\
-0.0784 \\
-0.0307 \\
0.000^{\circ} \\
0.000^{\circ}\end{array}$ & $\begin{array}{c}0.000 \% \\
-0.166 \% \\
-0.273 \% \\
- \\
- \\
\end{array}$ & $\begin{array}{r}0.2099 \\
47.5109 \\
11.2095 \\
0.0000^{\circ} \\
-0.365^{\circ} \\
\end{array}$ & $\begin{array}{r}0.2099 \\
0.2609 \\
-0.0405 \\
0.000^{\circ} \\
-0.365^{\circ} \\
\end{array}$ & $\begin{array}{c}1.866 \% \\
0.552 \% \\
-0.360 \% \\
- \\
-\end{array}$ \\
\hline \multicolumn{8}{|c|}{ ICA Feature Results for Cylinder 2: } \\
\hline Feature & True Value & $\begin{array}{c}\text { Sim. } \\
\text { Result }\end{array}$ & $\begin{array}{l}\text { abs.Sim. } \\
\text { Error }\end{array}$ & $\begin{array}{c}\text { rel.Sim. } \\
\text { Error }\end{array}$ & $\begin{array}{l}\text { Exp. } \\
\text { Result }\end{array}$ & $\begin{array}{c}\text { abs.Exp. } \\
\text { Error }\end{array}$ & $\begin{array}{c}\text { rel.Exp. } \\
\text { Error }\end{array}$ \\
\hline $\begin{array}{c}y \\
z \\
\text { radius } \\
\theta_{\mathrm{y}} \\
\theta_{\mathrm{z}} \\
\end{array}$ & $\begin{array}{r}-5.0000 \\
56.2500 \\
11.2500 \\
0.000^{\circ} \\
11.500^{\circ} \\
\end{array}$ & $\begin{array}{r}-4.9753 \\
56.2379 \\
11.2536 \\
0.599^{\circ} \\
11.385^{\circ} \\
\end{array}$ & $\begin{array}{r}0.0247 \\
-0.0121 \\
0.0036 \\
0.599^{\circ} \\
-0.115^{\circ} \\
\end{array}$ & $\begin{array}{c}0.219 \% \\
-0.021 \% \\
0.032 \% \\
- \\
-0.996 \%\end{array}$ & $\begin{array}{r}-4.4207 \\
55.7866 \\
11.2381 \\
-0.0912^{\circ} \\
10.876^{\circ} \\
\end{array}$ & $\begin{array}{r}0.5793 \\
-0.4634 \\
-0.0119 \\
-0.091^{\circ} \\
-0.624^{\circ} \\
\end{array}$ & $\begin{array}{c}5.149 \% \\
-0.824 \% \\
-0.106 \% \\
- \\
-5.423 \% \\
\end{array}$ \\
\hline \multicolumn{8}{|c|}{ ICA Feature Results for Cylinder 3: } \\
\hline Feature & True Value & $\begin{array}{c}\text { Sim. } \\
\text { Result }\end{array}$ & $\begin{array}{c}\text { abs.Sim. } \\
\text { Error }\end{array}$ & $\begin{array}{l}\text { rel.Sim. } \\
\text { Error }\end{array}$ & $\begin{array}{l}\text { Exp. } \\
\text { Result }\end{array}$ & $\begin{array}{c}\text { abs.Exp. } \\
\text { Error }\end{array}$ & $\begin{array}{c}\text { rel.Exp. } \\
\text { Error }\end{array}$ \\
\hline $\begin{array}{c}y \\
z \\
\text { radius } \\
\theta_{\mathrm{y}} \\
\theta_{\mathrm{z}}\end{array}$ & $\begin{array}{r}3.0000 \\
36.0500 \\
3.0500 \\
0.000^{\circ} \\
0.000^{\circ}\end{array}$ & $\begin{array}{r}3.0099 \\
36.0850 \\
3.0524 \\
-0.597^{\circ} \\
-0.051^{\circ}\end{array}$ & $\begin{array}{r}0.0099 \\
0.0350 \\
0.0024 \\
-0.597^{\circ} \\
-0.051^{\circ}\end{array}$ & $\begin{array}{c}0.325 \% \\
0.097 \% \\
0.077 \% \\
- \\
-\end{array}$ & $\begin{array}{r}3.0322 \\
35.9848 \\
2.8629 \\
2.8143^{\circ} \\
-0.122^{\circ}\end{array}$ & $\begin{array}{r}0.0322 \\
-0.0652 \\
-0.1871 \\
2.814^{\circ} \\
-0.122^{\circ}\end{array}$ & $\begin{array}{c}1.055 \% \\
-0.181 \% \\
-6.133 \% \\
- \\
-\end{array}$ \\
\hline
\end{tabular}

